\title{
Clasificación supervisada de imágenes PNOA-NIR y fusión con datos LiDAR-PNOA como apoyo en el inventario forestal. Caso de estudio: Dehesas
}

Supervised classification of PNOA-NIR images and datafusion with LiDAR-PNOA as support in a forest inventory: Case of study: Tree-grass environment "Dehesas"

Fragoso-Campón, L. ${ }^{* 1}$; Quirós, E. ${ }^{1}$; Gutiérrez Gallego, J.A. ${ }^{1}$

${ }^{1}$ Departamento de Expresión Gráfica, Universidad de Extremadura,

Escuela Politécnica, Avda. de la Universidads/n, 10003 Cáceres 


\title{
Resumen
}

La estimación de las métricas dasométricas como apoyo en un inventario forestal se puede abordar mediante la aplicación de distintas tecnologías y, si bien el muestreo de campo es la técnica más extendida, la aparición y desarrollo de las técnicas de teledetección aumentan las posibilidades de actuación en este ámbito. Estas nuevas técnicas de teledetección permiten minimizar los costes tanto económicos como en tiempo de la adquisición de datos sin menosprecio de la exactitud de las mediciones realizadas. En este sentido, las ortofotos del PNOA-NIR, que incluyen la información espectral del infrarrojo cercano, permiten obtener distintos índices de vegetación y suelo, aportando información valiosa en el análisis de la vegetación. En este trabajo se realiza una clasificación supervisada de las imágenes PNOA-NIR para, junto con los datos LiDAR-PNOA, determinar mediciones dendométricas en dehesas (área de copa, diámetro de copa y altura máxima de arbolado) y otros parámetros relacionados con la espesura (fracción de cabida cubierta y densidad). La metodología se plantea mediante la aplicación exclusivamente de software de código abierto. A través de los resultados obtenidos es posible delimitar de forma detallada la estructura de la copa, así como la reducción de la incertidumbre en las zonas de sombra del arbolado. La evaluación del clasificador empleado, Random Forest, alcanza un porcentaje de predicciones correctas del $96.72 \%$ con una confianza media en la clasificación de los píxeles de arbolado del 93\%. Por tanto, el método propuesto resulta adecuado para su aplicación en dehesas y otras masas abiertas sin tangencia de copas.

Palabras clave: dasometría, dendometría, QGIS, SNAP (Sentinel Application Platform), Random Forest.

\begin{abstract}
Obtaining dasometric attributes as support in a forest inventory can be addressed through the application of different technologies and, although field sampling is the most widespread technique, the current development of remote sensing techniques offer new opportunities. These new techniques make it possible to minimize both economic and time-related costs of acquiring data without disregarding the accuracy of the measurements made. In this sense, the orthophotos of the PNOA-NIR, which include near infrared spectral information, allow us to obtain different indices of vegetation and soil, providing valuable information in the analysis of the vegetation. In this work a supervised classification of the PNOA-NIR images is carried out and a data fusion with the LiDAR-PNOA data is made, to obtain dendometric measurements on tree-grass environments (dehesas) (crown area, crown diameter and maximum tree height) and other parameters related to the canopy spatial distribution (tree canopy cover factor and density). The methodology is based on the application of open source software exclusively. The obtained results delineate the structure of the crown in detail, reducing the uncertainty of the crown perimeter in shaded areas The evaluation of the classifier used, Random Forest (RF), reaches a percentage of correct predictions of $96.72 \%$ and an average confidence when classifying tree pixels of $93 \%$. Therefore, the proposed method is suitable for application in tree-grass environments and other forest areas without tree crown tangency.
\end{abstract}

Keywords: dasometry, dendrometry, SNAP (Sentinel Application Platform), Random Forest, QGIS. 


\section{Introducción}

La estimación de las métricas dasométricas en un inventario forestal se puede abordar mediante la aplicación de distintas tecnologías, en concreto, para la medición de árboles y masas forestales puede llevarse a cabo mediante la técnica tradicional de muestreo en campo, así como con técnicas fotogramétricas y mediante el uso de la teledetección. Si bien el muestreo de campo es la técnica más extendida, la aparición y el desarrollo actual de las técnicas de teledetección ofrecen nuevas oportunidades (Ortiz-Reyes et al., 2015).

Los datos de muestreo en campo son en muchos casos la única fuente de datos de las investigaciones (Diallo et al., 2013), pero también se utilizan como muestras de entrenamiento y validación en caso de aplicación de otras tecnologías de medición indirecta a través de métodos estadísticos de regresión (Uzquiano Pérez, 2014; Ortiz-Reyes et al., 2015; Guerra-Hernández et al., 2016). Entre las técnicas de teledetección, existen distintas tecnologías que principalmente se han centrado en estudiar áreas de tamaño reducido, como por técnicas fotogramétricas (Panagiotidis et al., 2017), con el LiDAR terrestre (Lin,Herold, 2016; López-Cortés et al., 2019) y small-foot print LiDAR (Popescu et al., 2004). Sin embargo, el uso del LiDAR aerotransportado a mayor escala (Popescu et al., 2003; Koukoulas,Blackburn, 2005; Falkowski et al., 2006) se ha convertido en los últimos años en una herramienta alternativa en mediciones de masas forestales (Uzquiano Pérez, 2014), que sirve de apoyo en posteriores análisis de regresión para la modelación de la relación entre variables dasométricas (Cancino, 2012; Navarro et al., 2018), permitiendo obtener la estructura tridimensional y continua de la masa forestal útil para completar los trabajos de campo (Palop-Navarro et al., 2016).

A la hora de plantear las mediciones dasométricas para un inventario forestal, entran en conflicto dos aspectos claves, por un lado, la exactitud de la información estructural detallada y, por otro, el coste económico y temporal que conlleva obtener esta información. La tendencia actual es la de minimizar los costes en tiempo y esfuerzo del trabajo de campo, pero sin menoscabar la calidad y exactitud de las mediciones. En este sentido, el uso tanto de información de acceso libre, así como su tratamiento posterior en software de código abierto (open source) permiten reducir los costes del inventario. En este sentido, España cuenta con la cobertura continua en todo el territorio del Plan Nacional de Ortofotografía Aérea (PNOA) que ofrece dos productos: imágenes orto rectificadas PNOA y nube de puntos LiDAR PNOA.

En primer lugar, imágenes orto-rectificadas se actualizan cada 2 o 3 años según las zonas y obtenidas como resultado del vuelo fotogramétrico realizado con una cámara digital multiespectral (Ministerio de Fomento, 2019). Estas imágenes están disponibles en dos formatos: PNOA-RGB, que incluyen la información espectral de las bandas visibles, y PNOA-NIR, que incluyen la información del infrarrojo cercano (NIR), rojo y verde. Los datos de la banda NIR complementan a los del espectro visible (RGB), y junto a ellos, permiten obtener distintos índices de vegetación y suelo, aportando información valiosa en el análisis de la vegetación. 
Por otro lado, ofrece la cobertura de puntos $3 \mathrm{D}$ tomada con sensor LiDAR aerotransportado, cuya densidad de puntos es de 0.5 puntos $\cdot \mathrm{m}^{-2}$ en la primera cobertura (vuelos de 2008 a 2010) y de 1 punto $/ \mathrm{m}^{-2}$ en la segunda cobertura (vuelos de 2016 a 2018).

La aplicación de los datos LiDAR-PNOA en mediciones forestales ha sido ya validada en distintos trabajos. En González-Ferreiro et al. (2012) se aplica en el estudio del $P$. radiata en Galicia concluyendo que es una buena fuente de información para reducir costes en el inventario forestal. Otro trabajo como el de Sánchez A1berola et al. (2018) aplica los datos en el análisis de la especie Pinus sylvestris L. y concluye igualmente que se consiguieron estimar con buena precisión distintas variables forestales indicando que la tecnología LiDAR tiene un gran potencial en la realización de inventarios forestales incluso utilizando datos de baja densidad como es el caso del LiDAR-PNOA. En Palop-Navarro et al. (2016) se cuantifica los estados sucesionales avanzados en bosques caducifolios del norte de la Península Ibérica tomando como base el INF4 y el vuelo LiDAR-PNOA, concluyendo que la cobertura LiDAR de baja densidad de puntos, puede identificar estructuras forestales relacionadas con bosques caducifolios maduros. No obstante, la aplicación de los datos LiDAR PNOA con baja densidad de puntos en zonas forestales con arbolado disperso es complicada ya que dificulta la posibilidad de tener puntos suficientes para identificar de forma detallada el dosel arbóreo (Sánchez Alberola et al., 2018). Este aspecto se agudiza aún más en las áreas de dehesas en las que la espesura del arbolado no supera el 60\% (Pulido,Picardo, 2010).

Si bien el uso de los datos LiDAR-PNOA está más extendido, el uso de la ortofoto del PNOA no es tan común en la realización de inventarios forestales. En Lavado Contador et al. (2012) se analiza la dinámica temporal de la cobertura del arbolado en dehesas mediante clasificaciones de imágenes PNOA-RBG. En BorlafMena et al. (2019) se utilizan de manera conjunta las imágenes PNOA-RGB y los datos LiDAR-PNOA para la extracción de la cobertura arbórea. En Navarro et al. (2018) se propone el uso de las imágenes PNOA-RGB para generar modelos digitales de la vegetación (MDV) por técnicas fotogramétricas, obteniendo buenos resultados siempre que exista un modelo digital del terreno (MDT) adecuado para poder normalizar las métricas de la vegetación.

En otros casos, el uso de las imágenes PNOA-RGB se reduce a la fotointerpretación de distintas coberturas o estimación de la fracción de cabida cubierta mediante el uso de platillas de densidad (Castillejo-González et al., 2010).

Respecto al uso de las imágenes PNOA-NIR, hay una notable ausencia de trabajos que las utilicen siendo uno de los motivos que no sean de libre acceso y solo están disponibles previa solicitud al Instituto Geográfico Nacional u organismo autonómico competente en la materia. Sin embargo, en Fragoso-Campón, Laura, y Quirós (2019) se analiza cómo la información espectral de la imagen PNOA-NIR puede resultar muy útil para la caracterización de las masas forestales en paisajes de dehesa.

Ante esta situación, el objetivo general del presente trabajo es evaluar la aplicación de las imágenes PNOA-NIR junto con los datos LiDAR-PNOA en la deter- 
minación de mediciones dendométricas en dehesas. Estas mediciones contemplan la extracción del perímetro de copa, medición del área y diámetro de copa, así como la estimación de la altura máxima de arbolado, y finalmente la aplicación en la determinación de otros parámetros dasométricos relacionados con la espesura de la masa como son la fracción de cabida cubierta y densidad del arbolado.

\section{Material y métodos}

\section{1. Área de estudio}

El análisis se ha llevado a cabo dentro del ámbito de la hoja del Mapa Topográfico Nacional a escala 1:50 000 (MTN50) n 752 ubicada en la Comunidad Autónoma de Extremadura. En concreto, el área corresponde a la cuadrícula a escala 1:5000 con número 752-8-5 (Fig. 1). El área se ubica dentro de la cuenca hidrográfica del río Aljucén caracterizada por un paisaje típico de la dehesa extremeña que, según la clasificación del Mapa Forestal de Extremadura, corresponde a encinar (Quercus rotundifolia).
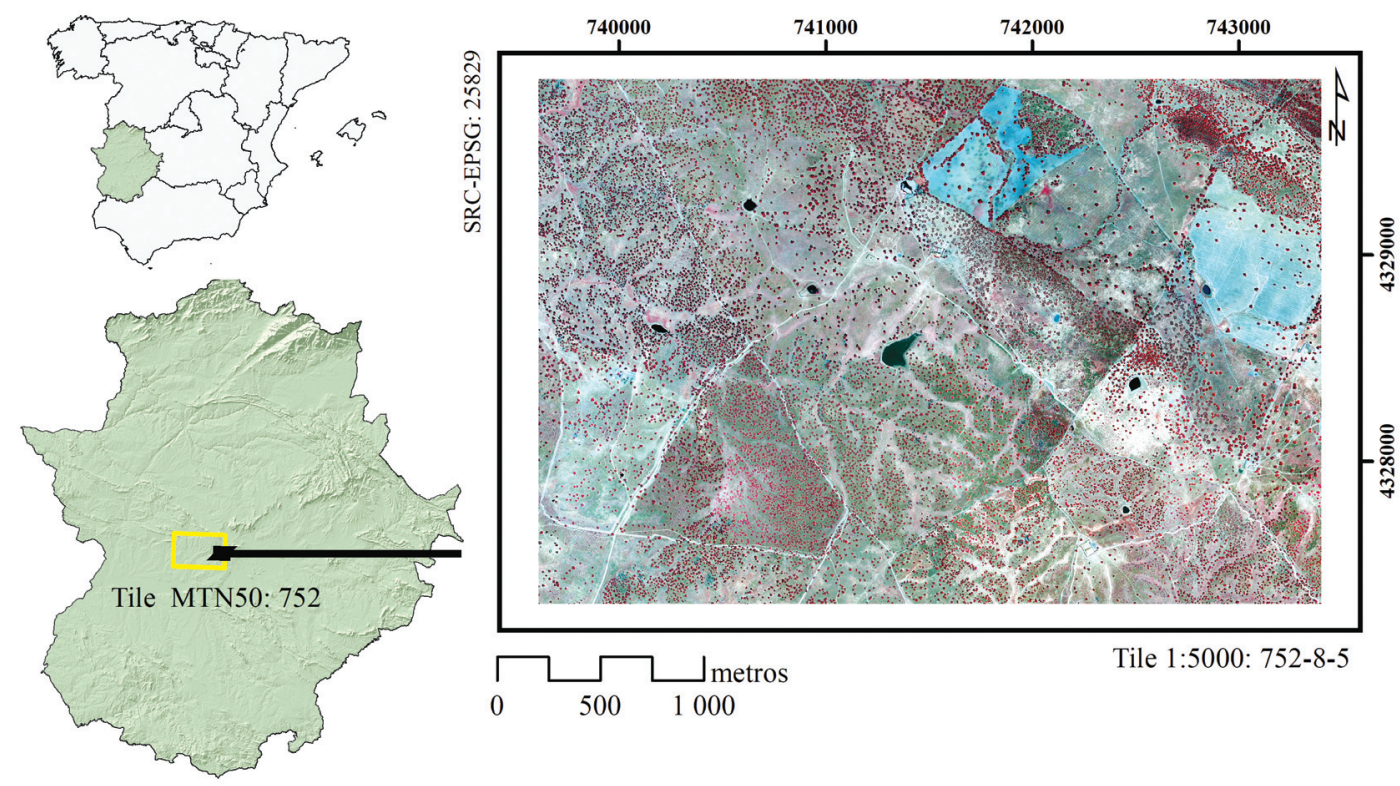

Figura 1. Delimitación de la zona de estudio. Hoja MTN50 no 752-8-5.

\subsection{Datos del Plan Nacional de Ortofotografía Aérea (PNOA)}

La imagen utilizada en este trabajo corresponde al vuelo de 2016 del Plan Nacional de Ortofotografía Aérea (PNOA) que presenta una resolución espacial de 
$0.25 \mathrm{~m}$. La imagen procede de un vuelo fotogramétrico realizado con una cámara digital de alta resolución, equipada con sensor pancromático y 4 sensores multiespectrales (Ministerio de Fomento, 2019). En concreto, se ha utilizado la ortofoto NIR que cuenta con las tres bandas correspondientes al infrarrojo cercano, rojo y verde, y que han sido facilitadas por el Centro de Información Cartográfica y Territorial de Extremadura (CICTEX). El vuelo disponible es de la época estival (junio de 2016) coincidiendo con la fase fenológica de la vegetación dominante en la que se observa una mayor separabilidad espectral entre la copa de arbolado y los estratos inferiores (Fragoso-Campón, Laura et al., 2019).

Los datos LiDAR en el área de estudio corresponden al vuelo disponible del 2010 y abarcan la extensión de seis cuadrículas (EXT 738-4328 EXT 738-4330, EXT 740-4328, EXT 740-43, EXT 742-4328, EXT 742-4330). La información se ha procesado con ayuda del software FUSION desarrollado por el Forest Service del USDA (McGaughey, 2009), generando como información final el MDV, en el que se representan las alturas de vegetación normalizadas con una resolución espacial de $1.5 \mathrm{~m}$ que es la mejor que se puede obtener considerando la baja densidad de puntos del vuelo LiDAR. Para ello, en primer lugar, se unieron todas las cuadrículas del vuelo LiDAR y, posteriormente, se eliminaron los registros atípicos y se extrajeron los retornos del suelo. Ésta es la nube de puntos que sirve para generar el MDT calculando la elevación de cada celda como promedio de la elevación de todos los puntos dentro de la celda. Posteriormente, se genera el MDV que representa la altura normalizada de la vegetación sobre el MDT.

\subsection{Procesado de la imagen}

La imagen se ha procesado con el programa Sentinel Application Platform (SNAP) desarrollado por la Agencia Europea Espacial (ESA) siguiendo la metodología expuesta en Fragoso-Campón, Laura, y Quirós (2019). La Fig. 3 muestra esquemáticamente el flujo de trabajo propuesto para el procesado de la imagen.

La información espectral disponible en la imagen PNOA se reduce únicamente a las tres bandas del NIR, rojo, verde y para completar la información puramente espectral, se han calculado varios índices de vegetación que faciliten la discriminación del estrato arbóreo como el NDVI, SAVI y MSAVI2; los índices de suelo BI y CI, así como el índice de agua NDWI (ver formulación en Tab. 1).

El análisis de las coberturas se ha realizado mediante una clasificación supervisada aplicando el algoritmo Random Forest (Breiman, 2001). Para ello se han definido las áreas de interés de manera aleatoria en el área de estudio, identificando las coberturas mediante fotointerpretación sobre la imagen, y finalmente se han considerado cinco clases: arbolado, pastizal, suelo desnudo y/o impermeable, masas de agua y sombras. Las áreas de interés se disponen en bloques de celdas de $1 \mathrm{~m}^{2}$ sobre las distintas coberturas, extrayendo de forma aleatoria el $75 \%$ para entrenamiento y el $25 \%$ para validación o evaluación de la exactitud. 
Tabla 1. Formulación de los índices de vegetación y suelos empleados.

\begin{tabular}{cl}
\hline Índice & Ecuación \\
\hline Vegetación & $S D V I=\frac{\text { nir }- \text { red }}{\text { nir }+ \text { red }}$ \\
& MSAVI $=(1+L) \cdot \frac{\text { nir }- \text { red }}{\text { nir }+ \text { red }+L} * \frac{1}{2} \cdot\left[\left(2 \cdot(\right.\right.$ nir +1$\left.)-\sqrt{(2 n i r+1)^{2}-8(\text { nir }- \text { red })}\right]$ \\
Suelo & $B I=\sqrt{\frac{\text { red }^{2}+\text { green }}{2}}$ \\
& $C I=\frac{\text { red }- \text { green }}{\text { red }+ \text { green }}$ \\
Agua & $N D W I=\frac{\text { green }- \text { nir }}{\text { green }+ \text { nir }}$
\end{tabular}

* Formulación tradicional adaptada a las bandas de las imágenes. ** $\mathrm{L}=0,5$

Fuente: Tabla 1 de Fragoso-Campón y Quirós (2019).

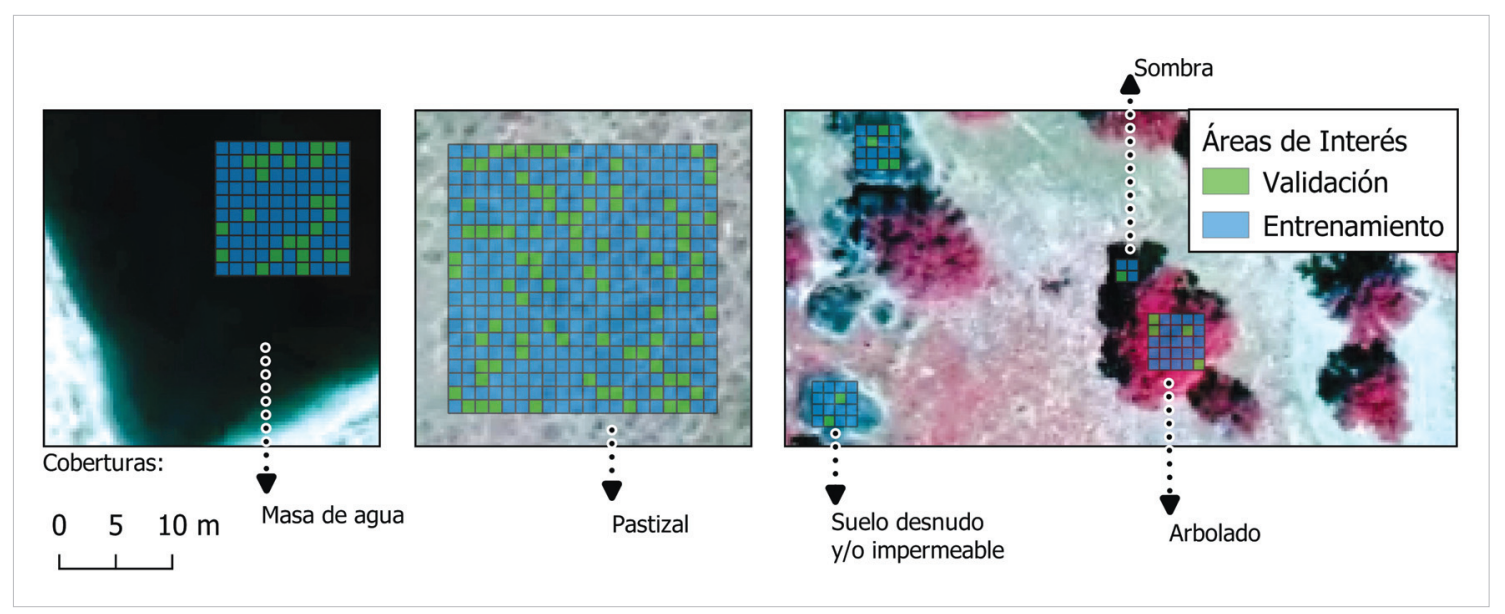

Figura 2. Áreas de entrenamiento y validación para evaluación de la exactitud.

La validación del mapa de coberturas obtenido se realiza mediante la validación cruzada del algoritmo de clasificación utilizado, así como con el mapa de confianza resultante en toda la extensión de la imagen obtenida.

\subsection{Post-Proceso de los resultados}

Una vez comprobada la validez del mapa ráster de la clasificación de coberturas, la siguiente fase se centra en obtener las variables dendométricas característi- 


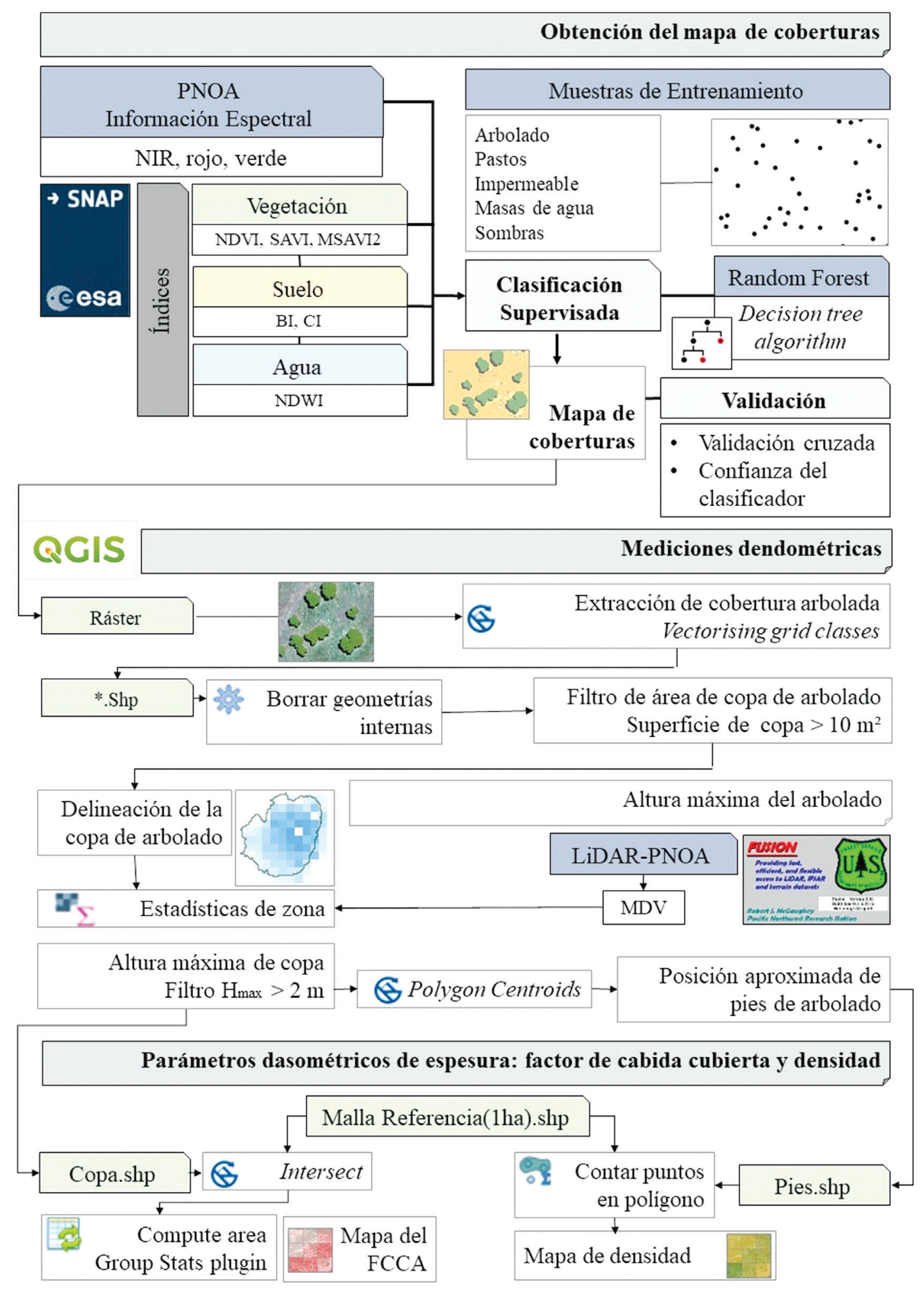

Figura 3. Metodología propuesta para el procesado de la imagen y el post-procesado de los resultados. 
cas como son: una delineación detallada de la copa del arbolado, superficie de la copa, diámetro de copa, y estimación de la altura máxima de la vegetación según el vuelo LiDAR. Finalmente se obtienen los parámetros dasométricos relacionados con la espesura de la masa como la fracción de cabida cubierta arbórea (Fcca) y densidad. Para ello, se realiza el postproceso de la imagen en QGIS aplicando distintas herramientas y algoritmos (ver el flujo de trabajo esquemático en la Fig. 3).

En primer lugar, se vectoriza el mapa ráster de coberturas indicándole al algoritmo que vectorice únicamente la cobertura que queremos analizar que es la del arbolado. En segundo lugar, se depuran las geometrías eliminando huecos interiores dentro de la copa inferiores a $1 \mathrm{~m}^{2}$, debidos a sombras superficiales por la rugosidad de la copa, y geometrías externas considerando un área mínima de copa de $10 \mathrm{~m}^{2}$. Una vez depurado el archivo vectorial que representa el arbolado, se obtienen las estadísticas de zona en cada copa tomando como referencia el ráster del MDV, seleccionando como copa del arbolado aquellos elementos con una altura máxima superior a 2 $\mathrm{m}$. Una vez depurado el archivo vectorial, se comprueba la exactitud de los resultados con la muestra independiente de evaluación obteniendo el porcentaje de acierto obtenido en la representación de las copas del arbolado. Finalmente, se obtiene la medición del área de copa y a partir de ella se estima su diámetro equivalente y la posición aproximada de los pies en el centro de gravedad de cada copa individual.

A partir de este punto, se define la malla $(100 \mathrm{~m}$ x $100 \mathrm{~m})$ en la que se van a calcular los estadísticos y posteriormente, se procede a obtener los parámetros de espesura de la masa forestal: el Fcca y la densidad (pies/ha) según se indica en la Fig. 3.

\section{Resultados y discusión}

Los resultados obtenidos en la clasificación supervisada de la imagen PNOA se muestran en las Fig. 4 que representa la clasificación de las distintas coberturas en la zona de estudio.

La validación cruzada del algoritmo de clasificación Random Forest muestra un porcentaje de predicciones correctas dentro de la muestra de entrenamiento del $96.72 \%$ con un $\mathrm{RMSE}=0.366$ y Bias $=-0.0049$. Los resultados obtenidos para cada cobertura se muestran en la Tab. 2, alcanzándose exactitudes entre el 0.97 y 1.

Tabla 2. Resultados obtenidos en la validación cruzada de las muestras de entrenamiento.

\begin{tabular}{cccccccc}
\hline Cobertura & Exactitud & VP & FP & VN & FN & Sensibilidad & Especificidad \\
\hline $\begin{array}{l}\text { Suelo desnudo } \\
\text { - impermeable }\end{array}$ & 100 & 996 & 5 & 3995 & 4 & 1.00 & 1.00 \\
Pastizal & 1.00 & 1000 & 0 & 4000 & 0 & 1.00 & 1.00 \\
Sombras & 0.97 & 925 & 86 & 3914 & 75 & 0.93 & 0.98 \\
Arbolado & 1.00 & 996 & 3 & 3997 & 4 & 1.00 & 1.00 \\
Masas de agua & 0.97 & 919 & 70 & 3930 & 81 & 0.92 & 0.98 \\
\hline
\end{tabular}




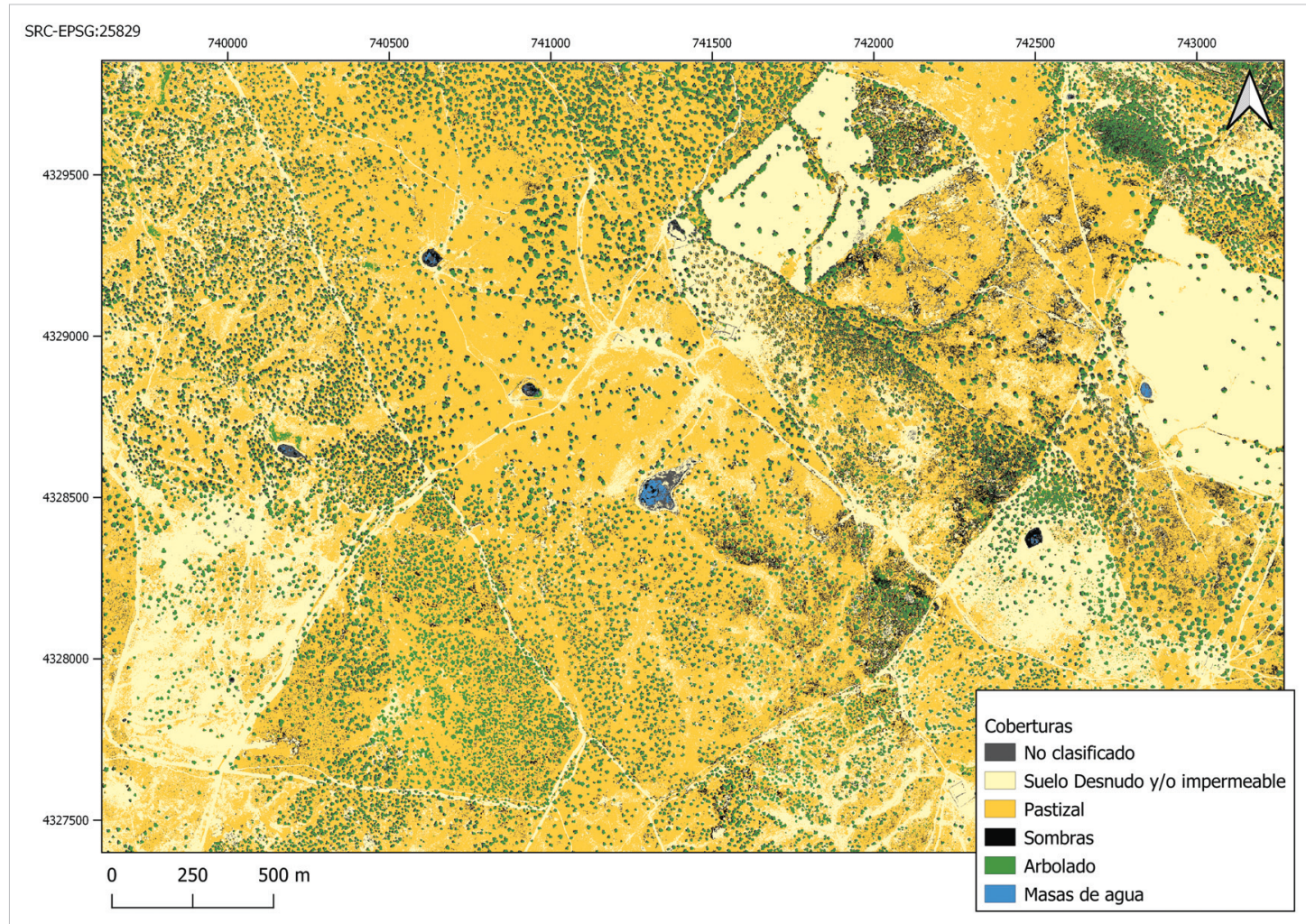

Figura 4. Mapa de coberturas.

Estos valores están en concordancia con los resultados obtenidos en Fragoso-Campón, Laura, y Quirós (2019) en otra área similar en la cuenca del río Tamuja en paisaje de dehesa.

Respecto a la confianza de la clasificación, la Fig. 5 muestra la confianza del clasificador en la asignación de la cobertura en cada píxel y la Tab. 3 muestra los valores medios de la confianza obtenidos en cada clase en toda el área de estudio.

Las coberturas de arbolado es la que se clasifica con mayor confianza, alcanzando un valor de confianza de 0.93 seguida de las coberturas de pastizal y suelo desnudo con un valor de 0.91 . Las coberturas con peor valoración son las sombras y masas de agua, que son las que presentan mayor confusión entre ellas, observándose un número de errores por falsos positivos y falsos negativos más numerosos (ver Tab. 2).

De los predictores utilizados en la clasificación, son las bandas del rojo y verde, junto con los índices de suelo BI y CI, los que cuentan con mayor poder predictivo, seguidos del NDWI, la banda del NIR, y los índices de vegetación SAVI y NDVI.

El control de la exactitud obtenido en la representación de las copas del arbolado tomando como referencia la muestra de validación del arbolado muestra un porcentaje de acierto del $97.56 \%$. Estos resultados están en concordancia con los 


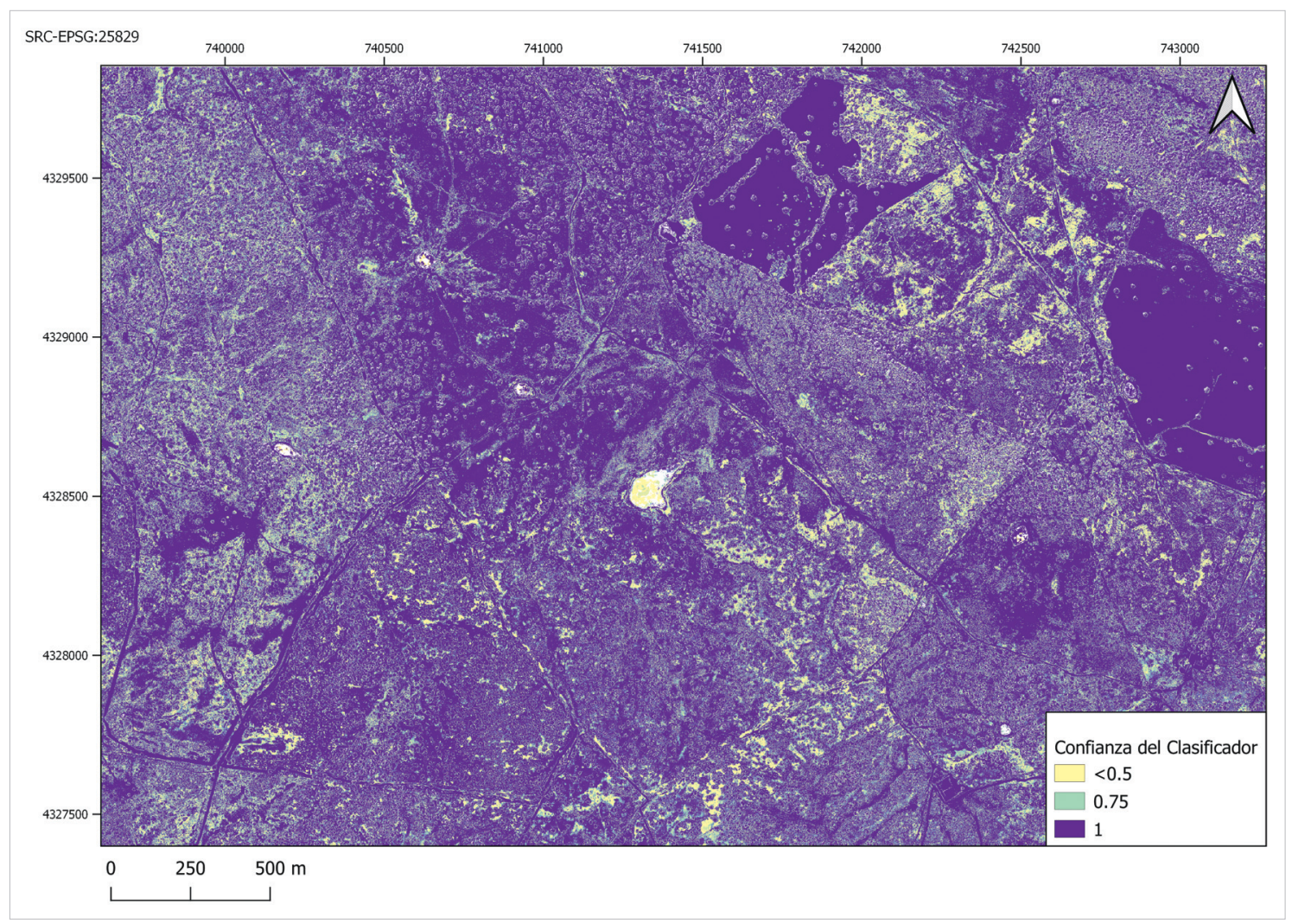

Figura 5. Confianza del clasificador.

Tabla 3. Confianza del clasificador Random Forest en la asignación de coberturas.

\begin{tabular}{lcc}
\hline \multicolumn{1}{c}{ Cobertura } & Valor medio & Desviación estándar \\
\hline Suelo desnudo y/o impermeable & 0.91 & 0.14 \\
Pastizal & 0.91 & 0.13 \\
Sombras & 0.79 & 0.20 \\
Arbolado & 0.93 & 0.14 \\
Masas de agua & 0.61 & 0.17 \\
\hline
\end{tabular}

obtenidos en Castillejo-González et al. (2010) donde se analiza la capacidad de discriminación de copas de árboles en una dehesa a partir de imágenes QuickBird mediante técnicas de combinaciones de bandas (RGB, NIR y NDVI) y su posterior aplicación en el cálculo de la Fcca. En este trabajo se obtienen unas fiabilidades globales entre el $80 \%$ y 98\%. Así mismo, los resultados obtenidos mejoran los resultados obtenidos de Borlaf-Mena et al. (2019) para la clasificación realizada en base a las imágenes PNOA-RGB, resaltándose la influencia positiva que ejerce en la clasificación la disponibilidad de la banda NIR, y la utilización de índices derivados. 
La evaluación de la exactitud y confianza obtenidas en la cobertura de arbolado ha permitido obtener con gran fiabilidad la delineación detallada de la copa. La Fig. 6 muestra el resultado final obtenido y representándose el dosel arbóreo en distintos rangos de altura según el dato de la altura máxima de la copa obtenida del vuelo LiDAR-PNOA.

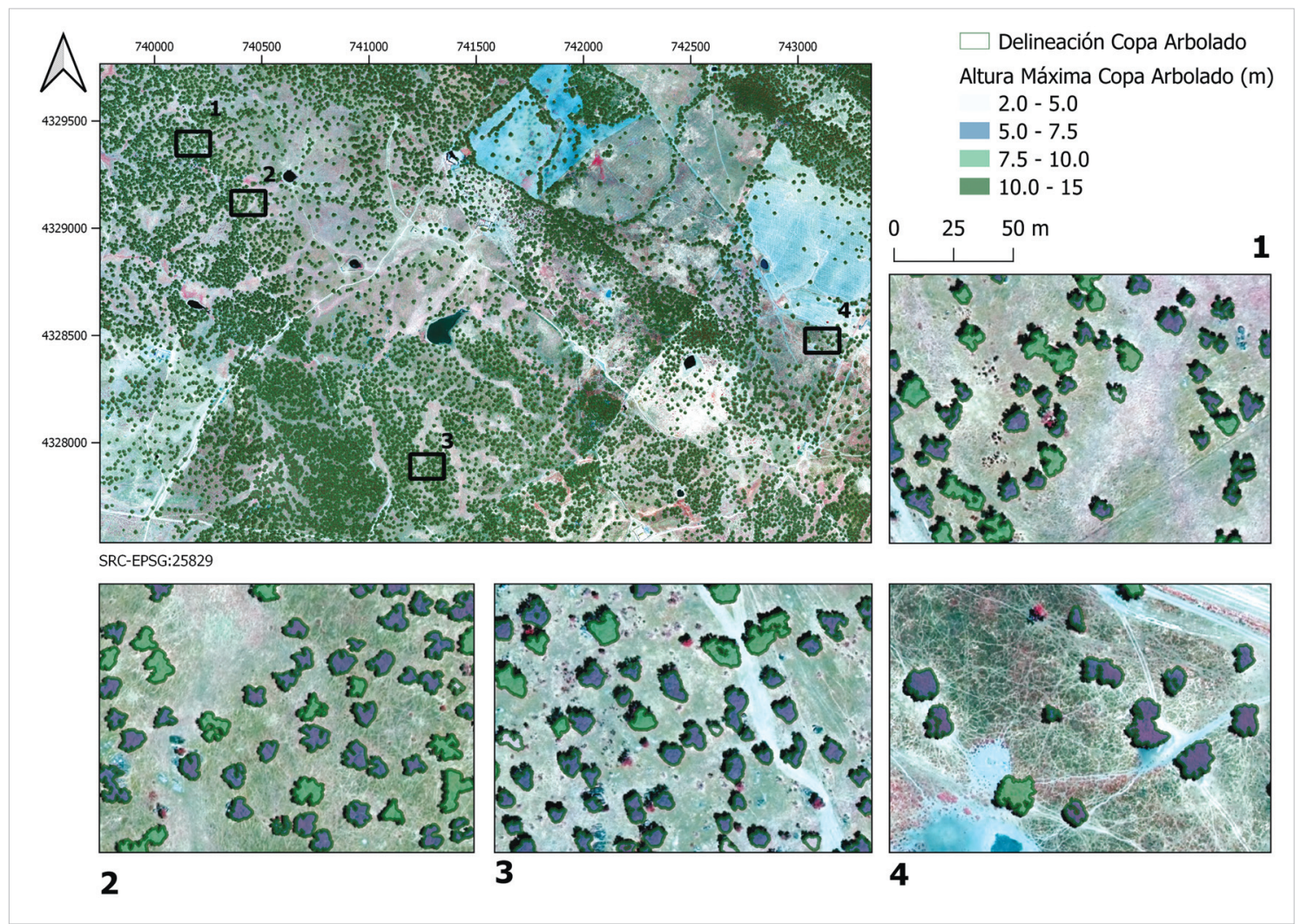

Figura 6. Mapa con la delineación detallada y altura máxima de la copa del arbolado.

El análisis visual de la delineación detallada de la copa del arbolado es más acertado que los resultados obtenidos en Cantero Fauquier et al. (2017) sobre todo la definición del perímetro y confusión en la zona de sombras. En Cantero Fauquier et al. (2017) se realiza una segmentación de la imagen PNOA-RGB y junto con datos derivados del modelo de altura procesados del LiDAR-PNOA, definen los contornos de copa. Si bien, no podemos cuantificar el margen de mejora, la clasificación supervisada de las imágenes PNOA-NIR junto con los predictores utilizados en el presente trabajo, como los índices de suelo y agua, mejoran la separabilidad de clases, permitiendo obtener mayor exactitud en la delineación del perímetro de copa, así como del diámetro de copa equivalente asignado a cada ejemplar de arbolado (Fig. 7).

Se han extraído las mediciones de todos los ejemplares detectados en la zona de estudio procesándose posteriormente en R (R-Core-Team, 2018) para obtener las 


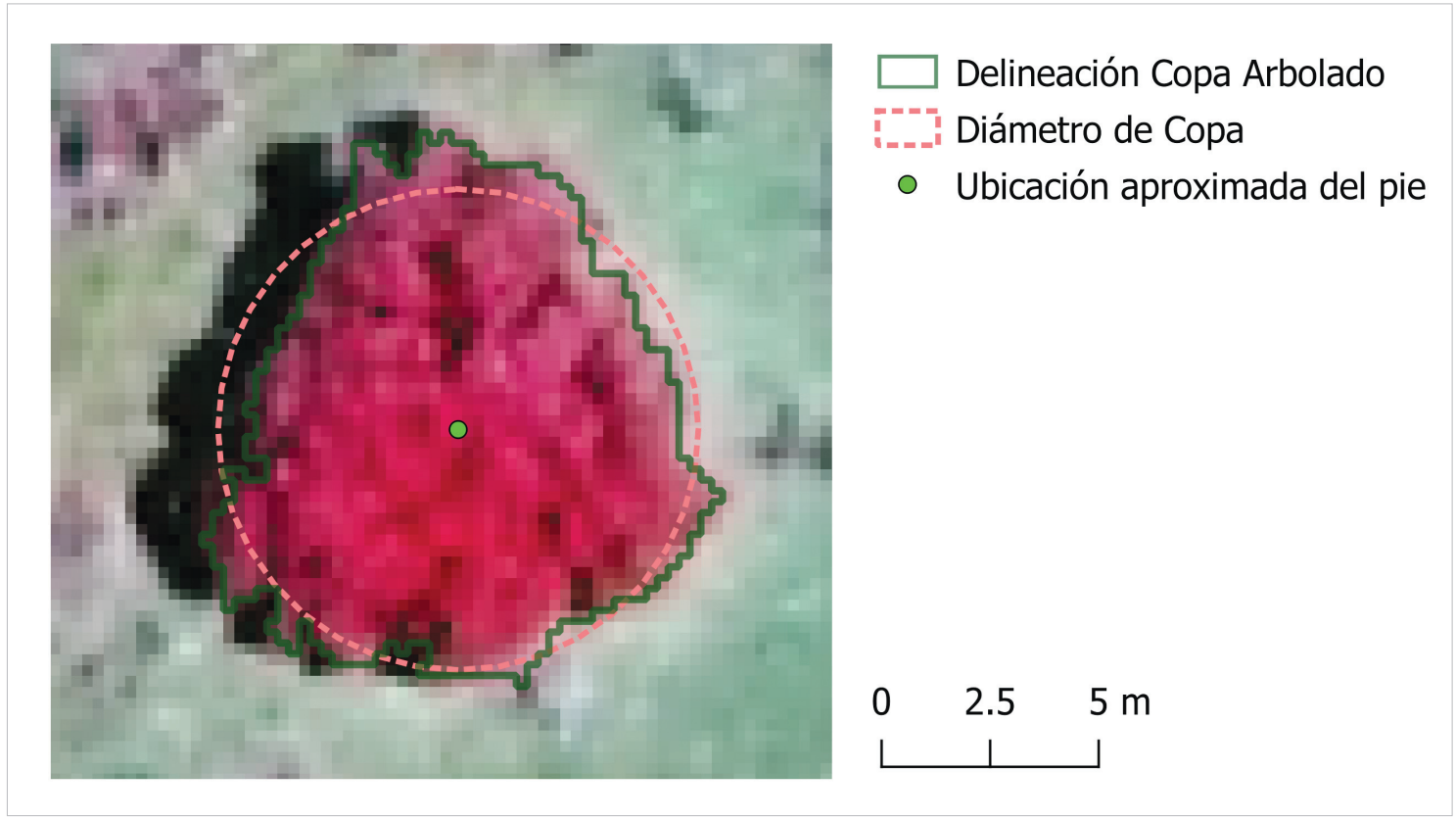

Figura 7. Detalle de la delineación detallada de la copa, el diámetro de copa equivalente y la ubicación aproximada del pie.

métricas representativas en el área estudiada. La Tab. 4 muestra el resumen de las métricas obtenidas del área y diámetro de copa, así como de la altura máxima obtenida del vuelo LiDAR-PNOA.

Tabla 4. Resumen de métricas del arbolado.

\begin{tabular}{lccc}
\hline \multicolumn{1}{c}{ Rango } & Área de copa $\left(\mathbf{m}^{2}\right)$ & Diámetro de copa $(\mathbf{m})$ & Altura Máxima $(\mathbf{m})$ \\
\hline Mínimo & 6.19 & 2.81 & 2.00 \\
ler cuartil & 28.19 & 5.99 & 5.17 \\
$2^{\circ}$ cuartil & 46.88 & 7.73 & 6.21 \\
3er cuartil & 69.75 & 9.42 & 7.21 \\
Máximo & 123.69 & 12.55 & 12.40 \\
\hline
\end{tabular}

En la Fig. 8 se presenta la caracterización de las alturas del arbolado en cada uno de los segmentos de percentil descritos en la Tab. 4.

Por otro lado, la Fig. 9 muestra la dispersión de la altura máxima de cada ejemplar y el diámetro equivalente de copa, así como los elipsoides de concentración para el 50\% y 90\% de los resultados, observándose que existe correlación positiva entre el tamaño de la copa y la altura máxima, presentando un coeficiente de correlación de Pearson de 0.55 . Se observa por tanto una tendencia similar a lo señalado en Diallo et al. (2013). Esta tendencia, permitiría establecer modelos de predicción en toda el área de estudio, si se completara la información disponible con 


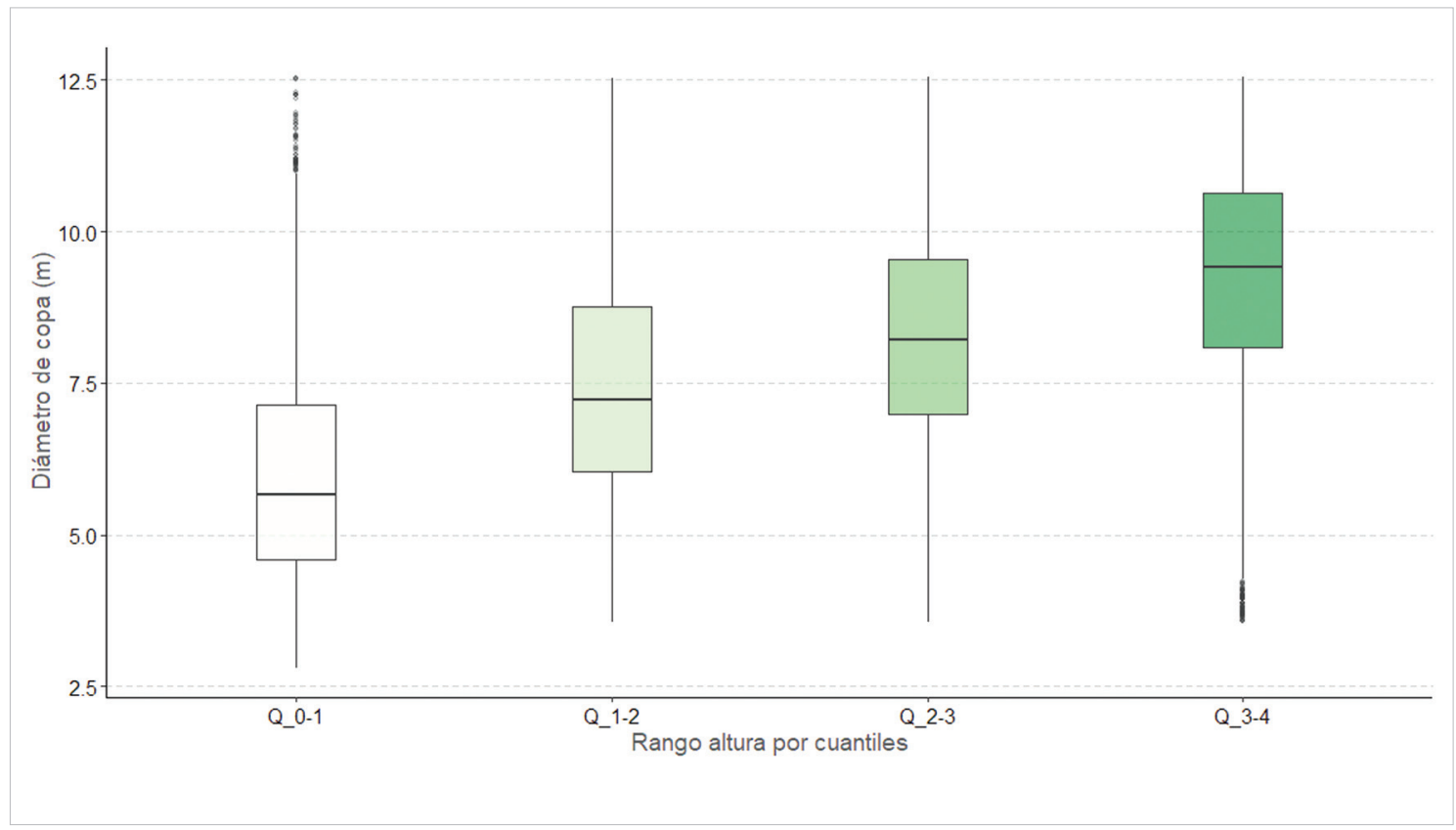

Figura 8. Distribución del diámetro de copas en cada rango de percentil de alturas.

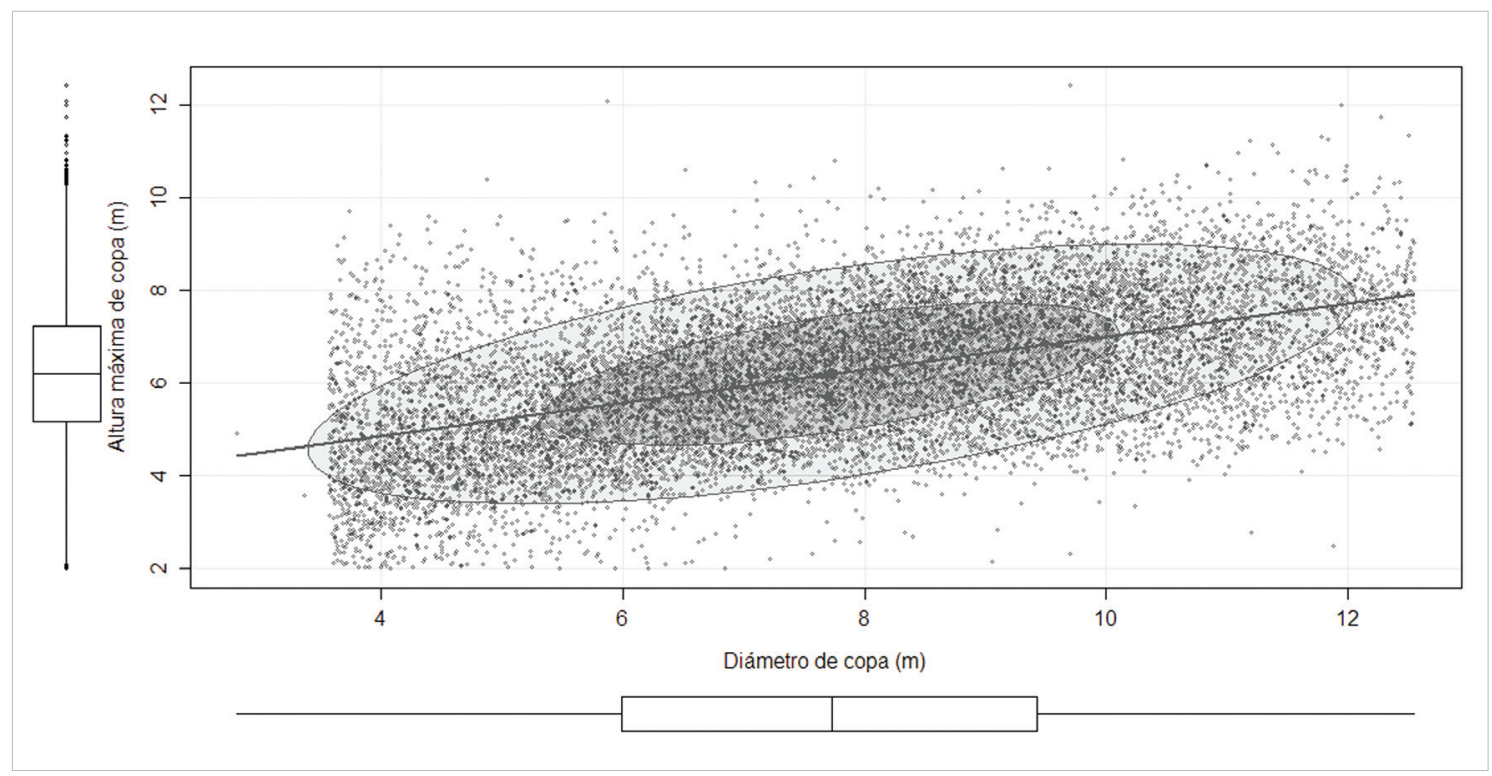

Figura 9. Diagrama de dispersión del diámetro de copa y la altura máxima de copa.

datos de campo considerando un muestreo representativo de otras variables dasométricas como el diámetro, área basimétrica, altura y volumen de copa o la estimación de la biomasa.

Por último, los resultados relacionados con la espesura se representan en la Fig. 10 y la Fig. 11 a través del mapa de la fracción de cabida cubierta y del mapa de densidad respectivamente. 


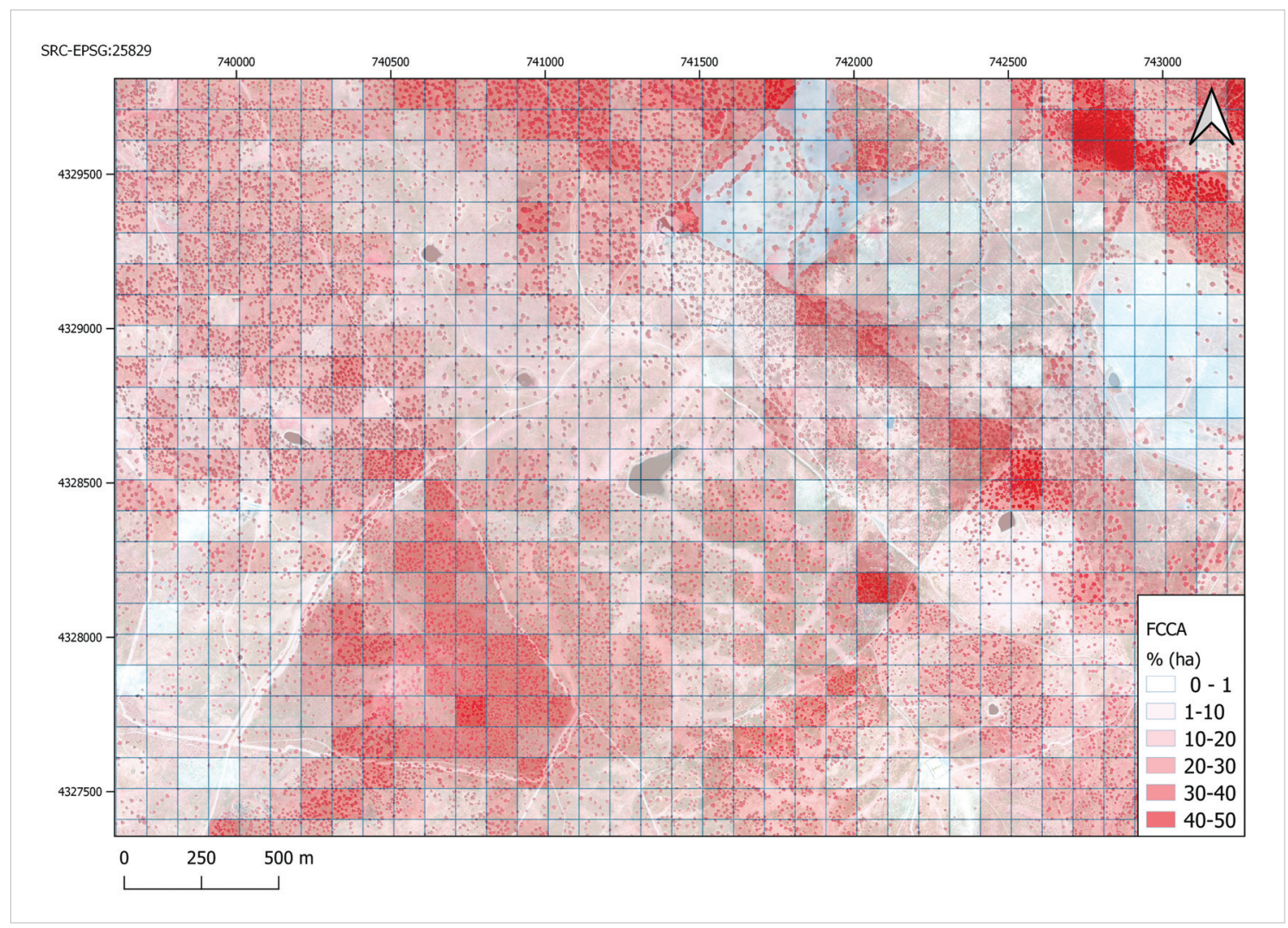

Figura 10. Mapa de la fracción de cabida cubierta arbórea (Fcca) por hectárea.

La mayor limitación de la metodología propuesta es la incertidumbre en la estimación de la densidad en las zonas en las que pueda existir tangencia de copas, sobre todo en las áreas con Fcca elevado. Al igual que se indica en Cantero Fauquier et al. (2017) la estimación de la densidad de pies y la identificación individual de las copas se complica en caso de tener alta cobertura y árboles con tangencia de copas, ya que una agrupación de distintos ejemplares estaría considerada como un único ejemplar y por tanto, la estimación de la densidad estaría infravalorada.

\section{Conclusiones}

En este trabajo se ha evaluado la aplicación de las imágenes PNOA-NIR junto con los datos LiDAR-PNOA en la determinación de mediciones dasométricas en dehesas. Se ha comprobado como la clasificación supervisada de las imágenes PNOANIR, basada en la información espectral y con ayuda de distintos índices de vegetación, suelo y agua derivados, permite la identificación detallada de cada copa individual del arbolado en zona de dehesa. La confianza de la clasificación supera el 93\% lo que garantiza la exactitud de las mediciones dendométricas relacionadas con la superficie de copa de cada ejemplar. Así, ha sido posible obtener tanto la me- 


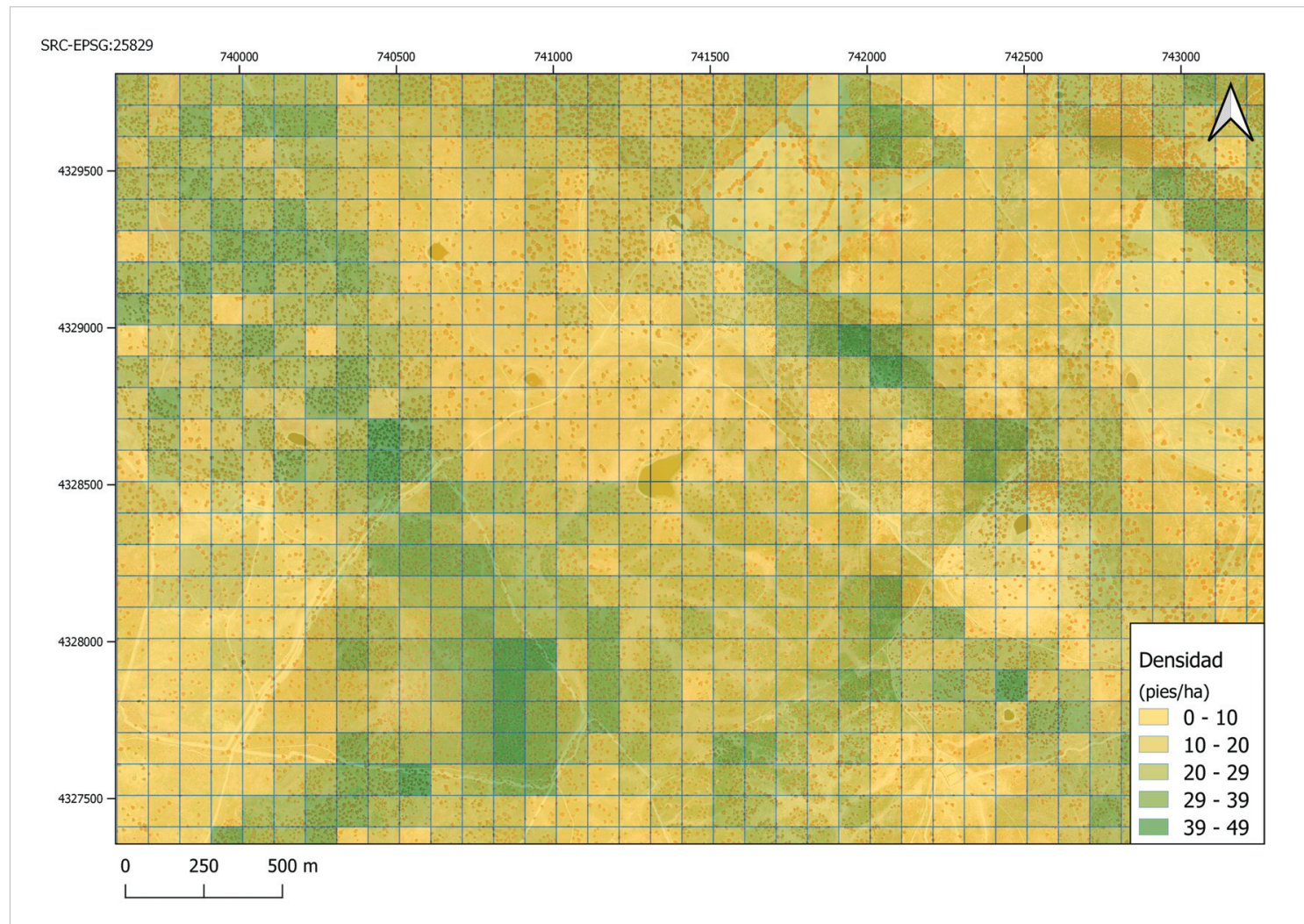

Figura 11. Mapa de la densidad del arbolado en pies por hectárea.

dición del área de copa y diámetro de copa, como otros parámetros dasométricos relacionados con la espesura de la masa como son la fracción de cabida cubierta y densidad del arbolado. Así mismo, la fusión de los resultados con la información derivada del vuelo LiDAR-PNOA ha permitido caracterizar la altura máxima de arbolado y estudiar la correlación existente con el resto de las variables en toda el área de estudio. Este proceso ha facilitado la caracterización de más de 13000 ejemplares. Todo el desarrollo metodológico se ha elaborado mediante la aplicación exclusivamente de software de código abierto. La principal limitación de la metodología propuesta de cara a su aplicación en futuros trabajos es la incertidumbre en la estimación de la densidad en las zonas en las que pudiera existir tangencia de copas.

\section{Agradecimientos}

La presente investigación se ha realizado gracias a la financiación de la Universidad de Extremadura y el Fondo Social Europeo: Una manera de hacer Europa, mediante una Ayuda para la Financiación de Contratos Predoctorales para la Formación de Doctores en los Centros Públicos de I+D pertenecientes al Sistema Extremeño de Ciencia, Tecnología e Innovación (Expediente PD16018). La difusión de este trabajo en las Jornadas de "Retos y Aplicaciones de los Inventarios Forestales" 
organizadas por la SECF ha sido posible también gracias a la ayuda del Fondo Europeo de Desarrollo Regional (FEDER) y Junta de Extremadura al grupo de investigación DESOSTE (GR18052). El CICTEX de la Junta de Extremadura ha proporcionado las imágenes del PNOA 2007 - CC-BY 4.0 scne.es.

\section{Bibliografía}

Borlaf-Mena, I.; Tanase, M.A.; Gómez-Sal, A.; 2019. Methods for tree cover extraction from high resolution orthophotos and airborne LiDAR scanning in Spanish dehesas. Revista de Teledetección, 53, 17-32. https://doi.org/10.4995/raet.2019.11320

Breiman, L.; 2001. Random forests. Machine learning. 45, 1, 5-32. https://doi.org/10.1023 /A:1010933404324

Cancino, J.; 2012. Dendrometría básica: Universidad de Concepción. Facultad de Ciencias Forestales. Departamento Manejo de Bosques y Medio Ambiente.

Cantero Fauquier, F.; Tomé Morán, J.; Bravo Fernández, J.; Fernández-Landa, A.; 2017. Herramientas de localización de árboles individuales con el módulo de procesado de QGIS a partir de información LiDAR y ortofotografía aérea. In:SECF (ed.) $7^{\circ}$ Congreso Forestal Español "Gestión del monte: servicios ambientales y bioeconomía". Plasencia, Cáceres (Spain).

Castillejo-González, I.L.; Medina Guerrero, J.; García-Ferrer Porras, A.; Mesas-Carrascosa, F.J.; Sánchez de la Orden, M.; 2010. Utilización de imágenes de satélite de alta resolución espacial en la determinación de la fracción de cabida cubierta en sistemas adehesados. In: Ojeda, J.; Pita, M.F.; Vallejo, I.; (ed.) XIV Congreso Nacional de Tecnologías de la Información Geográfica La información geográfica al servicio de los ciudadanos: de lo global a lo local. Sevilla (Spain).

Diallo, A.; Agbangba, E.C.; Ndiaye, O.; Guisse, A.; 2013. Ecological structure and prediction equations for estimating tree age, and dendometric parameters of Acacia senegal in the Senegalese semi-arid zone-Ferlo. American Journal of Plant Sciences. 4, 5, 1046. https://doi.org/10.4236/ajps.2013.45129

Falkowski, M.J.; Smith, A.M.; Hudak, A.T.; Gessler, P.E.; Vierling, L.A.; Crookston, N.L.; 2006. Automated estimation of individual conifer tree height and crown diameter via two-dimensional spatial wavelet analysis of lidar data. Canadian Journal of Remote Sensing. 32, 2, 153-161. https://doi.org/10.5589/m06-005

Fragoso-Campón, L.; Quirós, E.; 2019. Sentinel Toolbox Application (SNAP) aplicado a la clasificación supervisada de imágenes PNOA. In:UVA (ed.) XVIII Congreso de la Asociación Española de Teledetección: Hacia una visión global del cambio climático. Valladolid (España).

Fragoso-Campón, L.; Quirós, E.; Mora, J.; Gutiérrez Gallego, J.A.; Durán-Barroso, P.; 2019. Overstory-understory land cover mapping at the watershed scale: accuracy enhancement by multitemporal remote sensing analysis and LiDAR. Environmental Science and Pollution Research, 1-14. https://doi.org/10.1007/s11356-019-04520-8

González-Ferreiro, E.; Diéguez-Aranda, U.; Miranda, D.; 2012. Estimation of stand variables in Pinus radiata D. Don plantations using different LiDAR pulse densities. Forestry. 85, 2, 281-292. https://doi.org/10.1093/forestry/cps002 
Guerra-Hernández, J.; Tomé, M.; González-Ferreiro, E.; 2016. Cartografía de variables dasométricas en bosques Mediterráneos mediante análisis de los umbrales de altura e inventario a nivel de masa con datos LiDAR de baja resolución. Revista de Teledetección. 46, 103-117. https://doi.org/10.4995/raet.2016.3980

Koukoulas, S.; Blackburn, G.A.; 2005. Mapping individual tree location, height and species in broadleaved deciduous forest using airborne LIDAR and multi-spectral remotely sensed data. International Journal of Remote Sensing. 26, 3, 431-455. https://doi.org/ 10.1080/0143116042000298289

Lavado Contador, J.F.; Jariego García, A.; Schnabel, S.; Gómez Gutiérrez, Á.; 2012. Análisis de la evolución histórica del arbolado de la dehesa mediante fotointerpretación y análisis OBIA. In: Martínez Vega, J.; Martín Isabel, P.; (ed.) Tecnologías de la información geográfica en el contexto del cambio global: XV Congreso Nacional de Tecnologías de Información Geográfica. Madrid (Spain).

Lin, Y.; Herold, M.; 2016. Tree species classification based on explicit tree structure feature parameters derived from static terrestrial laser scanning data. Agricultural and Forest Meteorology. 216, 105-114. https://doi.org/10.1016/j.agrformet.2015.10.008

López-Cortés, I.; Martí-Gavilá, J.; Estornell, J.; Fernández-Sarría, A.; 2019. Comparación de parámetros de olivos a partir de UAV y datos LiDAR aéreos. In:UVA (ed.) XVIII Congreso de la Asociación Española de Teledetección: Hacia una visión global del cambio climático. Valladolid (España).

McGaughey, R.J.; (2009). FUSION/LDV: Software for LIDAR data analysis and visualization.

Ministerio de Fomento, 2019. Plan Nacional de Observación del Territorio. Plan Nacional de Ortofotografía Aerea (PNOA). http://pnoa.ign.es/presentacion Accesed. 22 abril 2019.

Navarro, J.A.; Fernández-Landa, A.; Tomé, J.L.; Guillén-Climent, M.L.; Ojeda, J.C.; 2018. Testing the quality of forest variable estimation using dense image matching: a comparison with airborne laser scanning in a Mediterranean pine forest. International Journal of Remote Sensing. 39, 14, 4744-4760. https://doi.org/10.1080/01431161.2018.147 1551

Ortiz-Reyes, A.D.; Valdez-Lazalde, J.R.; De-los-Santos-Posadas, H.M.; Ángeles-Pérez, G.; Paz-Pellat, F.; Martínez-Trinidad, T.; 2015. Inventario y cartografía de variables del bosque con datos derivados de LiDAR: comparación de métodos. Madera y bosques. 21, 3, 111-128. https://doi.org/10.21829/myb.2015.213461

Palop-Navarro, E.; Bañuelos, M.J.; Quevedo, M.; 2016. Combinando datos LiDAR e inventario forestal para identificar estados avanzadosde desarrollo en bosques caducifolios. Revista Ecosistemas. 25, 3, 35-42. https://doi.org/10.7818/ECOS.2016. 25-3.04

Panagiotidis, D.; Abdollahnejad, A.; Surový, P.; Chiteculo, V.; 2017. Determining tree height and crown diameter from high-resolution UAV imagery. International Journal of Remote Sensing. 38, 8-10, 2392-2410. https://doi.org/10.1080/01431161.2016.1264028

Popescu, S.C.; Wynne, R.H.; Nelson, R.F.; 2003. Measuring individual tree crown diameter with lidar and assessing its influence on estimating forest volume and biomass. Canadian Journal of Remote Sensing. 29, 5, 564-577. https://doi.org/10.5589/m03-027

Popescu, S.C.; Wynne, R.H.; Scrivani, J.A.; 2004. Fusion of small-footprint lidar and multispectral data to estimate plot-level volume and biomass in deciduous and pine forests 
in Virginia, USA. Forest Science. 50, 4, 551-565.

Pulido, F.; Picardo, A.; (2010). Libro verde de la dehesa. http:/www.eweb.unex.es/eweb/ac cionporladehesa/documentos/libro_verde_dehesa.pdf

R-Core-Team. (2018). R: A language and environment for statistical computing. In R. F. f. S. Computing (Ed.). Vienna (Austria).

Sánchez Alberola, J.; Oliver, P.; Estornell, J.; Dopazo, C.; 2018. Estimación de variables forestales de Pinus sylvestris L. en el contexto de un inventario forestal aplicando tecnología lidar aeroportada. GeoFocus. Revista Internacional de Ciencia y Tecnología de la Información Geográfica, 21, 79-99. https://doi.org/10.21138/GF.509

Uzquiano Pérez, S.; 2014. Mediciones Dendrométricas y Dasométricas mediante Técnicas LiDAR y Fotograméticas. Cuadernos de la Sociedad Española de Ciencias Forestales. 40, 193-202. https://doi.org/10.31167/csef.v0i40.17360 
